\title{
Effect of Isolated Anticonvulsant Drug Use and Associated to Midazolam as Pre-Anesthetic Medication on the Bispectral Index (BIS) in Patients with Cerebral Palsy
}

\author{
Verônica Vieira da Costa ${ }^{1}$, Renato Ângelo Saraiva, TSA ${ }^{2}$, Rafael Villela Derré Torres, TSA $^{3}$, \\ Sandro Barbosa de Oliveira ${ }^{4}$
}

\begin{abstract}
Summary: Costa VV, Saraiva RA, Torres RVD, Oliveira SB - Effect of Isolated Anticonvulsant Drug Use and Associated to Midazolam as PreAnesthetic Medication on the Bispectral Index (BIS) in Patients with Cerebral Palsy.

Background and objectives: Patients with cerebral palsy (CP) frequently receive drugs for the treatment of concomitant diseases, such as seizures. Midazolam is a benzodiazepine with hypnotic action most often used as pre-anesthetic medication and its drug interactions in patients with $\mathrm{CP}$ are unknown. The objective of the present study was to evaluate the effect of midazolam as pre-anesthetic drug on the BIS of patients with CP undergoing chronic treatment with anticonvulsant agents.
\end{abstract}

Method: Three groups of patients were assessed: CP without anticonvulsant treatment, CP undergoing treatment with anticonvulsant and a group with no disease and no medication use (control group). On the day before the surgery, with the patients conscious and in dorsal decubitus, the BIS monitor was placed and the basal BIS values were recorded. On the following day, 40 minutes before the surgery, the patients received 0.6 $\mathrm{mg} \cdot \mathrm{kg}^{-1}$ of midazolam orally. Before the start of the anesthetic procedure, the same procedure for BIS recording was carried out after midazolam administration.

Results: A total of 107 patients were studied - 39 patients from the Control Group (CG) and 68 with a diagnosis of CP. Among these, 17 used anticonvulsant drugs. Regarding the mean BIS value after the midazolam administration, there was no difference between patients from the CG and those in the CP group that did not take anticonvulsant drugs, whereas the ones who took anticonvulsants exhibited a difference $(p=0.003)$. The possibility of decrease in the BIS after midazolam use increases according to the number of anticonvulsant drugs used by the patient.

Conclusions: The chronic use of anticonvulsants associated to oral midazolam as pre-anesthetic medication can lead to the decrease in the BIS values, which configures deep level of hypnosis.

Keywords: DISEASES, Neurological: cerebral palsy; DRUGS: anticonvulsants; PRE-ANESTHETIC MEDICATION: midazolam; MONITORING: bispectral index.

[Rev Bras Anestesiol 2010;60(3): 259-267] @Elsevier Editora Ltda

\section{INTRODUCTION}

Cerebral palsy (CP) is a non-progressive disorder of posture and movement that is a consequence of cerebral injury, which can occur in the gestational, peri or post-natal periods ${ }^{1}$. It is the most common cause of motor dysfunction in children in developed countries and its prevalence among school aged children is estimated at approximately 2 in 1,000 live births, which has remained constant ${ }^{2}$.

Regardless of the etiological factor, patients with CP represent a challenge and need special care from anesthesiologists

Received from the Hospital Sarah, Brasilia, DF

1. Anesthesiologist of the Hospital Sarah; Master's degree in Rehabilitation Science

2. Coordinator of Anesthesiology of the Rede Sarah de Hospitais de Reabilitação; PhD in

Anesthesiology

3. Anesthesiologist of the Hospital Sarah

4. Statistician of the Hospital Sarah

Submitted on October 7, 2009

Approved on February 8, 2010

Correspondence to:

Dra. Verônica Vieira da Costa

SMHS, quadra 501, conj. A

70335-901-Brasília, DF, Brasil

E-mail:veve@sarah.br due to a series of factors. Among the clinical problems observed as part of the natural history of the disease is the unawareness of possible interactions with drugs commonly used by these patients and anesthetic agents ${ }^{3}$. Anticonvulsant agents are commonly used by patients with CP to control seizures, with carbamazepine and valproic acid being the most common. It is important for the CP patient to maintain these medications in the pre and postoperative periods to prevent seizures ${ }^{3}$.

Patients with CP are often admitted at hospitals for clinical or surgical treatments, which can generate a certain degree of anxiety. In spite of the multidisciplinary and psychological approach, sometimes it is necessary to use pre-anesthetic medication on the day before the surgery. Little is known on the combined effects of anticonvulsant agents and anesthetic drugs and doubts on the part of the anesthesiologist when faced with this situation. Is it necessary to decrease the dose of the pre-anesthetic medication? Will the patient present a deep degree of hypnosis?

The bispectral index (BIS) is a physiological variable used in the monitoring of anesthesia, which numerically expresses brain activity, being useful in the assessment of the degree of hypnosis during sedation and anesthesia ${ }^{4}$. Recent studies have demonstrated its validity in patients with $\mathrm{CP} 5$. 
The objective of the present study was to evaluate the effect of the chronic use of anticonvulsant agents on BIS monitoring, before and after the use of midazolam in patients with $\mathrm{CP}$.

\section{METHOD}

The study was approved by the Research Ethics Committee of the institution. The patients' participation was authorized by parents or tutors.

All patients aged 4 to 18 , of both sexes, who underwent an orthopedic surgical procedure from July 2005 to June 2006 were included in the sample. Patients who did not collaborate with the BIS monitoring, those with a history of paradoxical reaction to midazolam and patients from the Control Group (CG) that were receiving any other type of medication were excluded from the study.

Three groups of patients were studied prospectively: Group 1: patients with a diagnosis of CP defined according to the clinical criteria adopted by Rede Sarah de Hospitais de Reabilitação ${ }^{6}$ that used anticonvulsant agents; Group 2: patients with a diagnosis of CP that did not use anticonvulsant agents; Group 3: patients without central nervous system (CNS) and peripheral nervous system (PNS) diseases (Control Group).

The patients were being submitted to the surgical correction of orthopedic deformities and had been evaluated at the Outpatient Clinic of Anesthesia approximately 10 days before the surgical procedure. On the day before the surgery, during the pre-anesthetic visit at the infirmary, the basal BIS values were recorded with the patients conscious and in horizontal dorsal decubitus.

All patients received midazolam as pre-anesthetic medication 40 minutes before the surgery at a dose of $0.6 \mathrm{mg} /$ $\mathrm{kg}^{-1}$, orally. The patients from Group 1 (patients with a diagnosis of CP that used anticonvulsant drugs) also received the anticonvulsant agent in the morning before they were taken to the surgical center. The anticonvulsant agents used in association with others or not were: carbamazepine, vigabatrin, clonazepam, valproic acid, phenobarbital, phenytoin, lexotan, clobazam and lamotrigine.

Upon arrival at the anesthetic induction room, the patients were monitored through continuous electrocardiogram (ECG), noninvasive arterial pressure (NIBP), Saturation of Peripheral Oxygen $\left(\mathrm{SpO}_{2}\right)$ and subsequently a new BIS recording was obtained with the objective of comparing the basal BIS value with the value obtained after the midazolam administration.

The anesthesiologist that recorded the BIS value was blinded to whether the patients with CP used or not anticonvulsant drugs.

No clinical scale of sedation was used considering the common alterations in behavior and communication presented by patients with CP. Approximately $2 / 3$ of these patients present mental impairment and even those who do not present mental impairment can present attention deficit ${ }^{3}$, making it difficult to apply a clinical scale to evaluate the degree of sedation.
The descriptive analysis of the data was made at the statistical analysis. The Chi-square test was used to evaluate categorical variables and analysis of variance (ANOVA) to evaluate the continuous variables age and weight, considering a significance level of $p \leq 0.05$. The mean BIS value before and after the use of midazolam was assessed at four ranges: $100-90,90-80,80-60$ and $60-40$. The effect of the number of anticonvulsant drugs was also assessed, classified in two groups (patients that used only one anticonvulsant drug and those who used two or more drugs) on the mean BIS value. The logistic regression analysis was used to calculate the existence of the possibility of decrease in the BIS value after the use of midazolam in patients with $\mathrm{CP}$ that used anticonvulsant drugs.

\section{RESULTS}

A total of 107 patients of both sexes, aged 4 to 18 years, were studied. Of these, 68 had a diagnosis of CP and 39 were healthy patients, with no CNS or PNS diseases (CG). Of the 68 patients with $\mathrm{CP}, 51$ did not used anticonvulsant drugs and 17 did.

All clinical forms of CP were included in the group of patients with $\mathrm{CP}$, from the mild to the most severe forms (Table I).

Regarding the classification of the physical status (ASA), 25 patients from the CG were classified as PS-ASA I and 14 as PSASA II. Of the patients with CP that did not use anticonvulsant drugs, 8 were classified as PS-ASA I and 43 as PS-ASA II. Of the patients with $\mathrm{CP}$ that use $5 \mathrm{~d}$ anticonvulsant drugs, 13 were classified as PS-ASA II and 4 as PS-ASA III. There was a significant difference between the $C G$ and the PC group, as most patients in the CG had been classified in the PS-ASA I, whereas most patients from the PC group was classified as PS-ASA II and some as PS-ASA III $(p<0.001)$ (Table I). As for the distribution regarding age and sex, there was no difference between the groups.

As for the weight, there was a significant difference between the CP groups and the CG $(p=0.003)$. The mean weight in the $C G$ was higher than that in the PC group, regardless of the use of anticonvulsant drugs. There was no difference in the mean weight between the CP groups with and without anticonvulsant drugs.

As for the mean basal BIS value, there was no difference between patients from the $C G$ and the patients from the PC that did not use anticonvulsant drugs, whereas the patients with CP that used anticonvulsant drugs were different from the other groups $(p=0.003)$.

The mean BIS value after midazolam decreased in all studied groups when compared to the mean basal group, with the mean value in the CP group that used anticonvulsant drugs lower than that in the other groups, presenting statistical significance $(p=0.003)$.

All patients from the CG presented a mean basal BIS value between 90-100. In the CP group that did not use anticonvulsant drugs, $98 \%$ of the patients presented a mean basal BIS value between 90 and 100 and the other $2 \%$, between 80 and 90 . In the CP group that used anticonvulsant drugs, $76 \%$ pre- 
Table I - Characteristics of the Sample

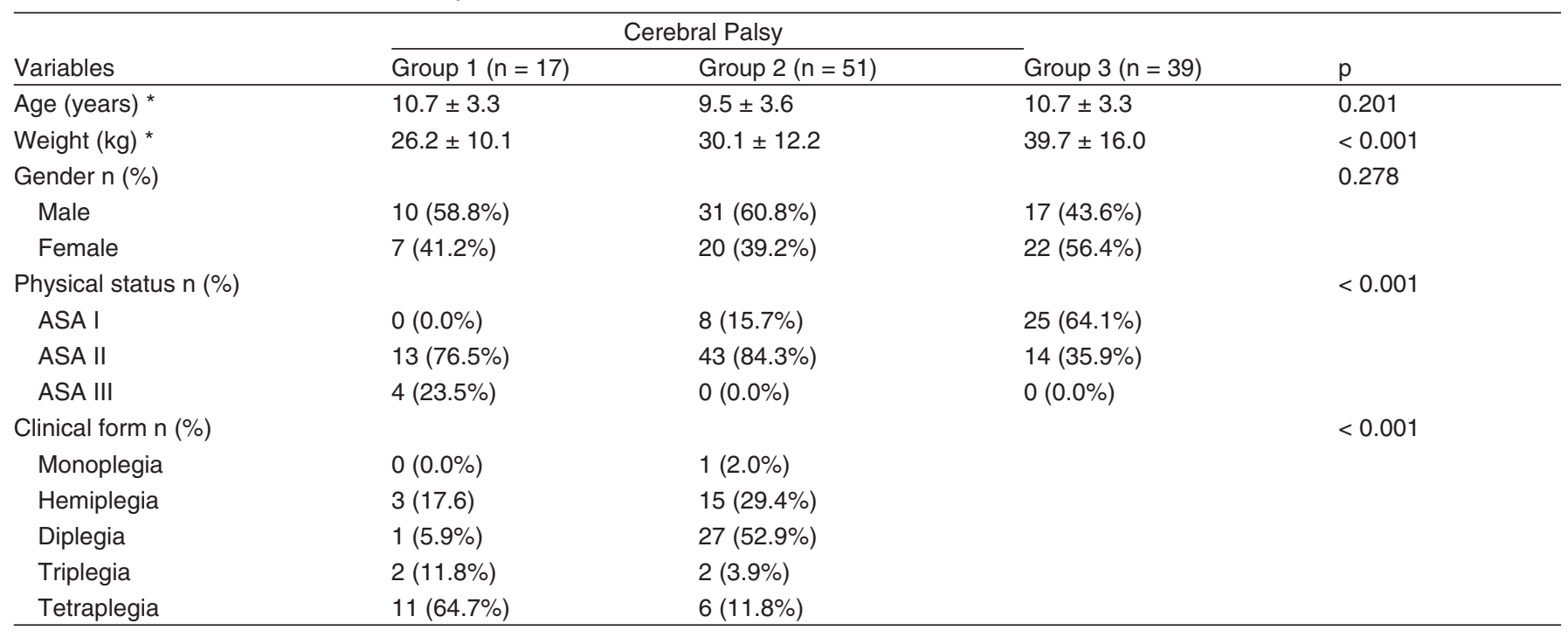

* Pearson's Chi-square

Group 1 - with anticonvulsant agent; Group 2 - without anticonvulsant agent; Group 3 - control.

sented a mean BIS value between 90 and $100,12 \%$ between $80-90$ and $12 \%$ between 40 and 60 (Table II).

In the CG, $21 \%$ of the patients presented alteration in the mean BIS value to the 80 to 90 range and $10.3 \%$ presented values in the 60 to 80 range. In the CP group that did not use anticonvulsant drugs, $12 \%$ presented alteration to the $80-90$ range and $14 \%$ presented alteration to the $60-80$ range. In the CP group that used anticonvulsant drugs, $12 \%$ presented a mean BIS value of 80 to $90,18 \% 60$ to 80 and $18 \%$ presented a mean BIS value of 40 to 60 (Table II).

Nine patients from CP group that used anticonvulsant agents received only one drug and the eight patients received two or more drugs.

Of the patients with CP that did not use anticonvulsant drugs, only $1(1.9 \%)$ presented severe mental impairment, $16(31.4 \%)$ presented cognitive deficit and $34(66.7 \%)$ had preserved cognitive function. Among the patients with CP that used anticonvulsant drugs, 11 (64.7\%) presented severe mental impairment, 4 (23.5\%) presented cognitive deficit and $2(11.8 \%)$ had preserved cognitive function.

With the objective of evaluating the influence of the anticonvulsant drugs on the decrease in BIS values after midazolam administration in patients with $\mathrm{CP}$, regardless of the use of the anticonvulsant drugs, an isolated analysis of this group was carried out. Of the group of patients with CP that did not use anticonvulsant drugs, $94.1 \%$ had a mean BIS value $>76$, whereas $5.9 \%$ presented a BIS value $\leq 75$ after midazolam administration. Of the patients that received only one type of anticonvulsant drug, $77.8 \%$ presented a mean BIS value $>76$, whereas $22.2 \%$ presented a mean BIS value $\leq 75$. Of the patients that received two or more anticonvulsant drugs, $50 \%$ presented a mean BIS value $>76$. The higher the number of anticonvulsant drugs, the lower the BIS value after midazolam administration $(p=0.03)$. It can be observed that patients with $\mathrm{CP}$ that received two or more anticonvulsant drugs are different from patients with $\mathrm{CP}$ that did not receive any or received only one anticonvulsant drug, when the mean BIS value is compared after midazolam administration.

With the objective of assessing the risk of decrease in the mean BIS value after midazolam administration according to the number of anticonvulsant drugs used by the patients, a logistic regression analysis was carried out in the patients with $\mathrm{CP}$ that used anticonvulsant drugs. Each anticonvulsant drug that the patient receives results in a 4-fold increase in the risk of decreasing the BIS value after midazolam administration to less than 75 (odds ratio $=4.02 ; 95 \% \mathrm{Cl}$ : $(1.63-9.87) ; \mathrm{p}=0.002$ ).

Table II - Mean Bispectral Index (BIS) Values Before and After Midazolam Administration

\begin{tabular}{|c|c|c|c|c|c|c|}
\hline \multirow[b]{2}{*}{ BIS } & \multicolumn{4}{|c|}{ Cerebral Palsy } & \multirow[b]{2}{*}{ Group $3(n=39)$} & \\
\hline & Group $1(n=17)$ & & Group $2(n=51)$ & & & \\
\hline & Basal a,b & Post-midazolam d,e & Basal a,c & Post-midazolam d,f & Basal b,c & Post-midazolam e,f \\
\hline $100-90$ & $13(76 \%)$ & $9(53 \%)$ & $50(98 \%)$ & $27(53 \%)$ & $39(100 \%)$ & $27(69 \%)$ \\
\hline $90-80$ & $2(12 \%)$ & $2(12 \%)$ & $1(2 \%)$ & $17(33 \%)$ & $0(0 \%)$ & $8(21 \%)$ \\
\hline $80-60$ & $0(0 \%)$ & $3(18 \%)$ & $0(0 \%)$ & $7(14 \%)$ & $0(0 \%)$ & $4(10 \%)$ \\
\hline $60-40$ & $2(12 \%)$ & $3(18 \%)$ & $0(0 \%)$ & $0(0 \%)$ & $0(0 \%)$ & $0(0 \%)$ \\
\hline
\end{tabular}

Pearson's Chi-Square for group distribution according to BIS

${ }^{\mathrm{a}} \mathrm{p}=1.000 ;{ }^{\mathrm{b}} \mathrm{p}=0.006 ;{ }^{\mathrm{c}} \mathrm{p}=0.021 ;{ }^{\mathrm{d}} \mathrm{p}=0.291 ;{ }^{\mathrm{e}} \mathrm{p}=0.037 ;{ }^{\mathrm{f}} \mathrm{p}=0.012$. 


\section{DISCUSSION}

The groups were similar regarding age and sex. As for the physical status, the underlying disease justifies the classification of the patients with CP as PS-ASA II or III, considering that the association between brain injury and other clinical conditions such as gastroesophageal reflux disease (GERD), respiratory system alterations, joint deformities, seizures, behavioral alterations and mental impairment is a common occurrence ${ }^{7,8}$. In more severe cases, the patients do not ambulate and remain most of the time in dorsal decubitus, which favors the accumulation of pulmonary secretion. As all clinical forms of CP were included in the study, some were classified as PS-ASA III.

As for the difference in weight between the CP group (regardless of the use or not of anticonvulsant drugs) and the $\mathrm{CG}$, the existence of gastrointestinal diseases such as GERD and malabsorption syndrome in patients with $\mathrm{CP}$ results in a lower weight than that considered ideal for the age ${ }^{7}$.

The mean basal BIS value in the group of patients with $\mathrm{CP}$ that received anticonvulsant drugs was lower than that in the CP group that did not use anticonvulsant drugs and in the CG. In $12 \%$ of the patients from the first group, the mean basal BIS was $<60$, which can configure a state of deep hypnosis ${ }^{9}$. The recordings were made in the infirmary on the day before the surgery, with the patients conscious in horizontal dorsal decubitus and accompanied by the parents or tutors. Perhaps the fact that the data were collected in a calming environment with the presence of the parents or tutors favored the recording of the data. The different degrees of cognitive function involvement are described as an effect of anticonvulsant drugs, even when administered at therapeutic doses ${ }^{10}$. Sedation is also described as a side effect of some anticonvulsant drugs, among them, phenytoin, phenobarbital, valproic acid, clonazepam, topiramate and gabapentin ${ }^{10}$, which many times are used in association (polytherapy). The use of polytherapy is described as an alternative in cases of seizures that are difficult to control, which occur mainly in cases of tetraplegia ${ }^{11}$. Most $(76 \%)$ of the patients with CP in the present study were tetraplegic and $64.7 \%$ used anticonvulsant drugs, which might have favored the results. In some occasions, polytherapy was used, which associated 2 or 3 of the aforementioned drugs to control seizures. There is a probable synergism between the drugs used in the treatment, which can explain the mean basal BIS values of the patients that received anticonvulsant drugs.

After the use of midazolam as pre-anesthetic medication the mean BIS value decreased in all groups; in the group with $\mathrm{CP}$ that received anticonvulsant drugs this decrease was more significant. In this group, before the use of midazolam, $76 \%$ of the patients presented BIS values between 90 and 100. After the administration of midazolam, only $53 \%$ persisted with a BIS value between 90 and 100 and some patients presented values as low as 40 to 60 . As each patient is his or her own control, it is probable that this decrease was caused by the association between the anticonvulsant drug and midazolam. The analysis performed only in the group of patients with $\mathrm{CP}$ showed that the effect of midazolam in the patients that received anticonvulsant drugs was more significant than in the patients that had the same diagnosis, but did not receive anticonvulsant drugs, which leads us to conclude that the association between the anticonvulsant drugs and midazolam has an cumulative effect on the BIS value, as the percentage of patients that started to present progressively lower BIS values increased after the administration of midazolam. Another interesting finding is that in patients that received more than one anticonvulsant drug the BIS value after midazolam administration was even lower, demonstrated by the logistic regression analysis.

There is a report in the literature on the increased sensitivity of patients with CP to sevoflurane, but the author does not mention whether the patients used anticonvulsant drugs ${ }^{12}$. In another study, the authors studied the action of midazolam as pre-anesthetic medication in patients with CP and concluded that midazolam results in a mild sedation without configuring levels of hypnosis, but do not report on the use of anticonvulsant drugs ${ }^{13}$. It is difficult to compare the present study with others in the literature, as no studies have been published using this methodology, which would favor comparison. In fact, little is known on cerebral palsy and its anesthetic implications to date.

The study has some limitations such as the fact that it is not a randomized study. The study group had had a definitive diagnosis and it would not be possible to study it without this previous definition. We included in the study all clinical forms of CP which might have caused a great variability in BIS values, but it would not have been possible to study CP without including all clinical forms, which are part of our routine practice.

What we wish to emphasize is that the chronic use of anticonvulsant drugs leads to a decrease in the BIS values and when this type of drug is associated with midazolam at a

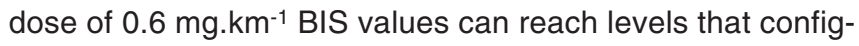
ure deep hypnosis. Patients with CP need multiple hospital admissions, which can cause them extreme anxiety, many times justifying the use of benzodiazepines as pre-anesthetic medication.

Considering the study conditions, it was possible to conclude that the chronic use of anticonvulsant drugs in patients with $\mathrm{CP}$, associated with the use of midazolam as pre-anesthetic medication resulted in a deeper level of hypnosis, and therefore, the risk-benefit analysis of this association must always be performed. 